\title{
US-Experten: Keine Sicherheitsbedenken bei inhalativen Kortikosteroiden
}

\section{Selbst an großen medizinischen Zentren mit breitem Einsatz von inhalativen Kortikosteroiden sind systemische Nebenwirkungen dieser Medikamentengruppe die Ausnahme. Dies ergab eine Umfrage unter mehr als 200 US-Asthmaexperten.}

S eit fast 20 Jahren werden inhalative Kortikosteroide bei Patienten mit persistierenden Asthmabeschwerden eingesetzt. Sie gelten inzwischen als Meilenstein in der präventiven antiinflammatorischen Behandlung. Obwohl in den multizentrischen Studien keine wesentlichen systemischen Nebenwirkungen gefunden wurden, ist ein Unbehagen geblieben. Dies dürfte mit den schlechten Erfahrungen zusammenhängen, die in der Anfangszeit der oralen Kortisonbehandlung gemacht wurden. Hier waren es vor allem die Auswirkungen auf die Hypothalamus-Hypophysen-NebennierenAchse (HHNA), welche das Kortison in Mißkredit brachten.

\section{Nebenwirkungen vor allem im Mundrachenraum}

In den letzten Jahren haben mehrere kleinere Studien auf eine mögliche Beeinflussung der HHNA auch durch inhalative Steroide hingewiesen. Um herauszufinden, ob diese Befunde im praktische Alltag tatsächlich relevant sind, verschickten William Storms und Charity Theen von den Asthma \& Allergy Associates in Colorado Springs 320 Fragebögen an die führenden Asthmaexperten Nordamerika. Die Responsquote von $67 \%$ ist außergewöhnlich hoch. Die Ärzte, zu 80\% Allergologen und zu 20\% Pneumologen, waren im Durchschnitt seit 16 Jahren ärztlich tätig und arbeiteten zur Zeit an Zentren der sekundären oder tertiären Versorgung.

Die Auswertung der Antworten zeigte, daß die meisten Nebenwirkungen von inhalativen Kortikoiden den Mundrachenraum betreffen. Heiserkeit, Halsschmerzen oder lokale Kandidainfektionen werden an den Zen- tren, an denen naturgemäß überwiegend die schwereren Fälle behandelt werden, von $48 \%$ der Ärzte ,gelegentlich“ gesehen. Als häufig wurden sie nur von 3\% der Spezialisten eingestuft. Die meisten Ärzte gaben an, daß diese Nebenwirkungen durch den Gebrauch von Spacern weitgehend vermieden werden kann. Zweithäufigste Nebenwirkung waren Hautveränderungen wie eine Neigung zu blauen Flecken oder eine Abnahme der Hautdicke. Sie wurden von $24 \%$ der Ärzte gelegentlich und von 6\% öfter bei den Patienten gesehen.

Weitere Nebenwirkungen waren eher die Ausnahme. Eine Gewichtszuaktualisiert worden. sollten.

Quelle: Reuters Health, August 1998 nahme wurde nur von $8 \%$ gelegentlich und von $1 \%$ häufig beobachtet. Veränderungen des Knochenstoffwechsels (Osteoporose und Frakturen bei Erwachsenen, Wachstumsprobleme bei Kindern) sahen über $80 \%$ der Ärzte nie bei ihren Patienten.

\section{„Epidemie“ von Kortisonschäden ist sicher auszuschließen}

Veränderungen der HHNA waren ebenfalls ungewöhnlich. Eine Senkung des Serumcholesterinwertes, eine gestörte ACTH-Stimulation sowie ein Hypoadrenalismus wurden nur von $1 \%$ der Ärzte als häufige Phänomene berichtet. Hierbei muß wiederum berücksichtigt werden, daß viele Patienten gerade an tertiären Zentren auch oral mit Kortikosteroiden behandelt werden. Daß eine „Epidemie" von Kortisonschäden durch diese Präparate bisher übersehen wurde, kann aufgrund der Umfrage jedoch ausgeschlossen werden.

(rme)

Quelle: Storms WW, Theen C: Clinical adverse effects of in haled corticosteroids: results of a questionnaire survey of asthma specialists. Ann Allergy Asthma Immunol 1998; 80

\section{FDA für striktere Warnhinweise bei inhalativen Kortikoiden}

Nach einer zweitägigen Anhörung von Experten hat die amerikanische Zulassungsbehörde Food and Drug Administration (FDA) striktere Warnhinweise für inhalative Kortikosteroide gefordert. Die Hersteller sollen in Zukunft verstärkt darauf hinweisen, daß es bei Kindern zu einer Verlangsamung des Körperwachstums kommen kann. Viele Medikamente trügen diese Hinweise schon, hieß es aus FDA-Kreisen, sie seien aber seit 15 Jahren nicht mehr

Jetzt sollen die Hersteller von Beclomethason-, Budesonid-, Flunisolid-, Fluticason- und Triamcinolon-haltigen Präparaten in den Beipackzetteln explizit darauf hinweisen, daß die Medikamente die Wachstumsgeschwindigkeit bei Kindern beeinträchtigen können und daß die Ärzte bei der Verordnung immer auch die Risiken für das Körperwachstum gegen den Nutzen abwägen

Eine Einschränkung der Indikation ist aber offenbar nicht geplant. Im Gegenteil: Im letzten Jahr hatte das National Heart, Lung and Blood Institute die Pädiater dazu aufgerufen, inhalative Steroide großzügiger einzusetzen. Viele Ärzte sind jedoch zurückhaltend, weil es noch nicht genügend Daten zum Einfluß der inhalativen Steroide auf das Wachstum gibt. Vier Firmen, Glaxo Wellcome, Astra, Schering-Plough und Rhône-Poulenc Rorer haben deshalb bei der FDA plazebokontrollierte Studien beantragt, welche mehr Gewißheit für die Nutzen-Risiko-Abschätzung geben sollen.

(red) 